\title{
PARECERISTAS AD HOC 2021
}

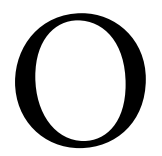

s Editores da revista Educação \& Sociedade e a Diretoria do Centro de Estudos Educação e Sociedade (Cedes) agradecem a colaboração de todos os membros de seu Conselho Editorial, colaboradores permanentes e, particularmente, aos pareceristas ad hoc elencados a seguir, que emitiram pareceres e promoveram, com sua experiência e profissionalismo, a arbitragem dos textos submetidos para avaliação neste periódico, durante o ano de 2021.

Adilson Dalben

Universidade Estadual de Campinas, Campinas (SP), Brasil

Adriana Lia Friszman Laplane

Universidade Estadual de Campinas, Campinas (SP), Brasil

Adriana Mabel Fresquet

Universidade Federal do Rio de Janeiro, Rio de Janeiro (RJ), Brasil

Adriana Russi

Universidade Federal Fluminense, Niterói (RJ), Brasil

Aislan Vieira de Melo

Instituto Federal de Mato Grosso do Sul, Campo Grande (MS), Brasil Alan Vitor Pimenta

Universidade Federal de São Carlos, São Carlos (SP), Brasil

Alberto Filipe Ribeiro de Abreu Araújo

Universidade do Minho, Minho, Portugal

Alda Regina Tognini Romaguera

Universidade de Sorocaba, Sorocaba (SP), Brasil

Alessandro de Melo

Universidade Estadual do Centro-Oeste, Guarapuava (PR), Brasil

Alessandro Soares da Silva

Universidade de São Paulo, São Paulo (SP), Brasil

Alexandra Bujokas de Siqueira

Universidade Federal do Triângulo Mineiro, Uberaba (MG), Brasil

Alexandre Filordi de Carvalho

Universidade Federal de São Paulo, São Paulo (SP), Brasil

Alexandre Rodrigo Nishiwaki da Silva

Universidade Federal de São Carlos, São Carlos (SP), Brasil

Alice Casimiro Lopes

Universidade Estadual do Rio de Janeiro, Rio de Janeiro (RJ), Brasil
Alicia Catalano Bonamino

Pontifícia Universidade Católica do Rio de Janeiro, Rio de Janeiro (RJ), Brasil

Altair Alberto Fávero

Universidade de Passo Fundo, Passo Fundo (RS), Brasil

Amarildo Luiz Trevisan

Universidade Federal de Santa Maria, Santa Maria (RS), Brasil

Amelia Cristina Abreu Artes

Fundação Carlos Chagas, São Paulo (SP), Brasil

Amélia Veiga

Universidade do Porto, Porto, Portugal

Amone Inácia Alves

Universidade Federal de Goiás, Goiânia (GO), Brasil

Ana Carmen Amorim Jara Casco

Universidade Federal Fluminense, Niterói (RJ), Brasil

Ana Clara Bortoleto Nery

Universidade Estadual Paulista Júlio de Mesquita Filho, Marília (SP), Brasil

Ana Crhistina Vanali

Universidade Tecnológica Federal do Paraná, Curitiba (PR), Brasil

Ana Laura Godinho Lima

Universidade de São Paulo, São Paulo (SP), Brasil

Ana Lúcia Silva Souza

Universidade Federal da Bahia, Salvador (BA), Brasil

Ana Luiza Bustamante Smolka

Universidade Estadual de Campinas, Campinas (SP), Brasil

Ana Maria Vieira

Instituto Politécnico de Leiria, Leiria, Portugal

Ana Paula de Oliveira Corti

Instituto Federal de Educação, Ciência e Tecnologia de São Paulo, São Paulo (SP), Brasil 
André Luiz Paulilo

Universidade Estadual de Campinas, Campinas (SP), Brasil

Andrea Barrientos Soto

Universidad de Granada, Granada, Espanha

Andrea Maila Voss Kominek

Universidade Tecnológica Federal do Paraná, Curitiba (PR), Brasil

\section{Andrea Moruzzi}

Universidade Federal de São Carlos, São Carlos (SP), Brasil

Andréa Lopes da Costa Vieira

Universidade Federal do Estado do Rio de Janeiro, Rio de Janeiro (RJ), Brasil

Andreia Barbosa Gouveia

Universidade Federal do Paraná, Curitiba (PR), Brasil

Andréia Ferreira da Silva

Universidade Federal de Campina Grande, Campina Grande (PB), Brasil

Angela Scalabrin Coutinho

Universidade Federal do Paraná, Curitiba (PR), Brasil

Ângelo Ricardo Souza

Universidade Federal do Paraná, Curitiba (PR), Brasil

Angelo Vitorio Cenci

Universidade de Passo Fundo, Passo Fundo (RS), Brasil

Antoni Verger

Universitat Autònoma de Barcelona, Barcelona, Espanha

\section{António Fragoso}

Universidade do Algarve, Faro, Portugal

António Magalhães

Universidade do Porto, Porto, Portugal

Antônio Carlos Dias Junior

Universidade Estadual de Campinas, Campinas (SP), Brasil

Antônio Lisboa Leitão

Universidade Federal de Campina Grande, Campina Grande (PB), Brasil

Aracy Martins

Universidade Federal de Minas Gerais, Belo Horizonte (MG), Brasil

Arlete Ramos dos Santos

Universidade Estadual de Santa Cruz, Ilhéus (BA), Brasil

Beatriz Barrero Fernández

Universidad de Granada, Granada, Espanha
Beatriz Gerherson

Pontifícia Universidade Católica do Rio Grande do Sul, Porto Alegre

(RS), Brasil

Belinda Mandelbaum

Universidade de São Paulo, São Paulo (SP), Brasil

Bernardete Angelina Gatti

Fundação Carlos Chagas, São Paulo (SP), Brasil

Breitner Tavares

Universidade de Brasília, Brasília (DF), Brasil

Carlos Alberto Feliciano

Universidade Estadual Paulista Júlio de Mesquita Filho, Presidente

Prudente (SP), Brasil

Carlos Eduardo Ribeiro

Universidade Federal do ABC, Santo André (SP), Brasil

Carmen Gloria Núñez Muñoz

Pontificia Universidad Católica de Valparaiso, Viña del Mar, Chile

Carmen Rodriguez Martinez

Universidad de Málaga, Málaga, Espanha

Carolina Mostaro

Universidade de São Paulo, São Paulo (SP), Brasil

Carolina Stuchi

Universidade Federal do ABC, Santo André (SP), Brasil

Cassia Domiciano

Universidade Federal do Paraná, Curitiba (PR), Brasil

Catarina de Almeida Santos

Universidade de Brasília, Brasília (DF), Brasil

Catarina Ianni Segatto

Universidade Federal do ABC, Santo André (SP), Brasil

Catarina Tomás

Escola Superior de Educação de Lisboa, Lisboa, Portugal

Cesar Teixeira

Universidade Federal do Rio de Janeiro, Rio de Janeiro (RJ), Brasil

Cidinalva Silva Câmara Neris

Universidade Federal do Maranhão, São Luís (MA), Brasil

Cláudia da Mota Darós Parente

Universidade Estadual Paulista Júlio de Mesquita Filho, Marília (SP), Brasil

Claudia Lemos Vóvio

Universidade Federal de São Paulo, São Paulo (SP), Brasil 
Cláudia Oliveira Pimenta

Rede Estadual de Ensino Paulista, São Paulo (SP), Brasil

\section{Cláudia Pereira Vianna}

Universidade de São Paulo, São Paulo (SP), Brasil

Claudia Regina Baukat Silveira Moreira

Universidade Positivo, Curitiba (PR), Brasil

Cláudia Valentina Assumpção Galian

Universidade de São Paulo, São Paulo (SP), Brasil

Claudio Duarte Quapper

Universidad de Chile, Santiago, Chile

Claudio Patricio Almonacid Aguila

Universidad de Chile, Santiago, Chile

\section{Cleber Vieira}

Universidade Federal de São Paulo, São Paulo (SP), Brasil

Cristiane Machado

Universidade Estadual de Campinas, Campinas (SP), Brasil

\section{Cristina Aracely Muñoz Moran}

Universidad Evangélica de El Salvador, San Salvador, El Salvador

Dagoberto Buim Arena

Universidade Estadual Paulista Júlio de Mesquita Filho, Marília (SP), Brasi

Dalila Andrade Oliveira

Universidade Federal de Minas Gerais, Belo Horizonte (MG), Brasil

Daniel Cara

Universidade de São Paulo, São Paulo (SP), Brasil

Daniel Fonseca de Andrade

Universidade do Estado do Rio de Janeiro, Rio de Janeiro (RJ), Brasil

Daniela de Oliveira Pires

Universidade Federal do Paraná, Curitiba (PR), Brasil

Darlan de Mamann Marchi

Universidade Federal de Pelotas, Pelotas (RS), Brasil

\section{Débora Dainez}

Universidade Estadual de Campinas, Campinas (SP), Brasil

Debora Goulart

Universidade Federal de São Paulo, São Paulo (SP), Brasil

\section{Débora Mazza}

Universidade Estadual de Campinas, Campinas (SP), Brasil

Deborah Christina Antunes

Universidade Federal do Ceará, Sobral (CE), Brasil
Denise Maria Penna Kronemberger

Fundação Instituto Brasileiro de Geografia e Estatística, Rio de Janeiro (RJ), Brasil

Domingo Barroso-Hurtado

Universidad de Granada, Granada, Espanha

Doriedson do Socorro Rodrigues

Universidade Federal do Pará, Cametá (PA), Brasil

Douglas Altamiro Consolo

Universidade Estadual Paulista Júlio de Mesquita Filho, São José do Rio Preto (SP), Brasil

Edison Alencar Casagranda

Universidade de Passo Fundo, Passo Fundo (RS), Brasil

Edivaldo Gois Junior

Universidade Estadual de Campinas, Campinas (SP), Brasil

Edmilson Antonio Pereira Junior

Universidade Federal de Minas Gerais, Belo Horizonte (MG), Brasil

\section{Eduardo Donizeti Girotto}

Universidade de São Paulo, São Paulo (SP), Brasil

Eduardo Pinto e Silva

Universidade Federal de São Carlos, São Carlos (SP), Brasil

Elaine Sampaio Araújo

Universidade de São Paulo, Ribeirão Preto (SP), Brasil

Eliane Aparecida da Silva

Instituto Federal de Educação, Ciência e Tecnologia de Mato Grosso,

Cuiabá (MT), Brasil

\section{Eliza Bartolozzi Ferreira}

Universidade Federal do Espírito Santo, Vitória (ES), Brasil

Elizabeth Aponte Jaramillo

Universidad Autònoma de Occidente Cali, Colômbia

Elizabeth Macedo

Universidade do Estado do Rio de Janeiro, Rio de Janeiro (RJ), Brasil

Elmir de Almeida

Universidade de São Paulo, São Paulo (SP), Brasil

Enicéia Gonçalves Mendes

Universidade Federal de São Carlos, São Carlos (SP), Brasil

\section{Ênio Serra}

Universidade Federal do Rio de Janeiro, Rio de Janeiro (RJ), Brasil

Enrique Baleriola Escudero

Universitat Oberta de Catalunya, Barcelona, Espanha 
Erika Moreira Martins

Universidade Estadual de Campinas, Campinas (SP), Brasil

Ernesto Seidl

Universidade Federal de Santa Catarina, Florianópolis (SC), Brasil

Eucidio Pimenta Arruda

Universidade Federal de Minas Gerais, Belo Horizonte (MG), Brasil

Eva González Ortega

Universidad de Salamanca, Salamanca, Espanha

Fabiana Augusta Alves Jardim

Universidade de São Paulo, São Paulo (SP), Brasil

Fabiana Marini Braga

Universidade Federal de São Carlos, São Carlos (SP), Brasil

Fabiana Silva Fernandes

Fundação Carlos Chagas, São Paulo (SP), Brasil

Fabio Luiz T. Crocco

Instituto Tecnológico de Aeronáutica, São José dos Campos (SP), Brasil

\section{Fátima Cabral}

Universidade Estadual Paulista Júlio de Mesquita Filho, Marília (SP), Brasil

Fátima Antunes

Universidade do Minho, Minho, Portugal

Felícia Silva Picanço

Universidade Federal do Rio de Janeiro, Rio de Janeiro (RJ), Brasil

Fernando Luiz Cássio

Universidade Federal do ABC, Santo André (SP), Brasil

Fernando Tavares Jr

Universidade Federal de Juiz de Fora, Juiz de Fora (MG), Brasil

Fernando Silva Teixeira-Filho

Universidade Estadual Paulista Júlio de Mesquita Filho, Assis (SP), Brasil

Flávia Mateus Rios

Universidade Federal de Goiás, Goiânia (GO), Brasil

Francisca Izabel Pereira Maciel

Universidade Federal de Minas Gerais, Belo Horizonte (MG), Brasil

\section{Francisco André Silva Martins}

Universidade do Estado de Minas Gerais, Belo Horizonte (MG), Brasil

\section{Gabriela Portugal}

Universidade de Aveiro, Aveiro, Portugal
Geraldo Balduino Horn

Universidade Federal do Paraná, Curitiba (PR), Brasil

Geraldo Leão

Universidade Federal de Minas Gerais, Belo Horizonte (MG), Brasil

Gionara Tauchen

Universidade Federal do Rio Grande

Giovanna Modé Magalhães

Universidade de São Paulo, São Paulo (SP), Brasil

Gisele Masson

Universidade Estadual de Ponta Grossa, Ponta Grossa (PR), Brasil

Giselle Cristina Martins Real

Universidade Federal da Grande Dourados, Dourados (MS), Brasil

Giselle Martins dos Santos Ferreira

Pontifícia Universidade Católica do Rio de Janeiro, Rio de Janeiro (RJ), Brasil

Gleicy Silva

Universidade Estadual de Campinas, Campinas (SP), Brasil

Graciela Carmen Muñoz Zamora

Universidad Metropolitana de Ciencias de la Educación, Ñuñoa, Chile

Graciela Morgade

Universidad de Buenos Aires, Buenos Aires, Argentina

Guilherme do Val Toledo Prado

Universidade Estadual de Campinas, Campinas (SP), Brasil

Guilherme Ramalho Arduini

Universidade Estadual de Campinas, Campinas (SP), Brasil

Guilherme Silva

Universidade do Minho, Minho, Portugal

Heitor Queiroz de Medeiros

Universidade Católica Dom Bosco, Campo Grande (MS), Brasil

Helena Costa Lopes de Freitas

Universidade Estadual de Campinas, Campinas (SP), Brasil

Henrique Tahan Novaes

Universidade Estadual Paulista Júlio de Mesquita Filho, Marília (SP), Brasil

Hermengarda Alves Ludke

Pontifícia Universidade do Rio de Janeiro, Rio de Janeiro (RJ), Brasil

Hivy Melo

Universidade Estadual de Campinas, Campinas (SP), Brasil 
Iana Gomes de Lima

Universidade da Região de Joinville. Joinville (SC), Brasil

Iraneide Soares da Silva

Universidade Estadual do Piauí, Teresina (PI), Brasil

Isis Silva Roza

Universidade Federal de Ouro Preto, Ouro Preto (MG), Brasil

Isaac Paxe

Instituto Superior de Ciências da Educação de Luanda, Luanda

Isaac Pipano

Universidade Estadual Paulista Júlio de Mesquita Filho, Bauru (SP), Brasil

Ivan Pincheira

Universidad de Chile, Santiago, Chile

Janete Flor de Maio

Universidade Federal de Ouro Preto, Ouro Preto (MG), Brasil

Jeanne Marie Gagnebin

Universidade Estadual de Campinas, Campinas (SP), Brasil

Jefferson Mainardes

Universidade Estadual de Ponta Grossa, Ponta Grossa (PR), Brasil

Jenny Assael

Universidad de Chile, Santiago, Chile

João Ferreira de Oliveira

Universidade Federal de Goiás, Goiânia (GO), Brasil

João Reis Silva Jr

Universidade Federal de São Carlos, São Carlos (SP), Brasil

Joaze Bernardino Costa

Universidade de Brasília, Brasília (DF), Brasil

Jonas Marcondes Sarubi de Medeiros

Centro Brasileiro de Análise e Planejamento, São Paulo (SP), Brasil

Jorge Adelino Costa

Universidade de Aveiro, Aveiro, Portugal

Jorge Ramos do Ó

Universidade de Lisboa, Lisboa, Portugal

Jorge Ruiz-Morales

Universidad de Sevilla, Sevilla, Espanha

José Antonio Pineda Alfonso

Universidad de Sevilla, Sevilla, Espanha

Jose Augusto Palhares

Universidade do Minho, Minho, Portugal
José Carlos Rothen

Universidade Federal de São Carlos, São Carlos (SP), Brasil

José Euzébio de Sousa Aragão

Universidade Estadual Paulista Júlio de Mesquita Filho, Rio Claro (SP), Brasil

José Licínio Backes

Universidade Católica Dom Bosco, Campo Grande (MS), Brasil

Jose Manuel Gonzalez

Universidad de Zaragoza, Zaragoza, Espanha

José Pedro Boufleuer

Universidade Regional do Noroeste do Estado do Rio Grande do Sul, Ijuí (RS), Brasil

Jose Renato Polli

Universidade Estadual de Campinas, Campinas (SP), Brasil

Jose-Javier Navarro-Perez

Universidad de Valencia, Valencia, Espanha

Joyce Mary Adam

Universidade Estadual Paulista Júlio de Mesquita Filho, Rio Claro (SP), Brasil

Judite Primo

Universidade Lusófona de Humanidades e Tecnologia, Lisboa, Portugal Juliana Silva Santos

Instituto Federal de Educação, Ciência e Tecnologia de Minas Gerais, Belo Horizonte (MG), Brasil

\section{Julvan Moreira Oliveira}

Universidade Federal de Juiz de Fora, Juiz de Fora (MG), Brasil

Justino Magalhães

Universidade de Lisboa, Lisboa, Portugal

Kátia Augusta Curado Pinheiro Cordeiro da Silva

Universidade de Brasília, Brasília (DF), Brasil

Kátia Evangelista Regis

Universidade Federal do Maranhão, São Luís (MA), Brasil

Katia Morosov Alonso

Universidade Federal de Mato Grosso, Cuiabá (MT), Brasil

Kátia Rubio

Universidade de São Paulo, São Paulo (SP), Brasil

Laura Ceretta Moreira

Universidade Federal do Paraná, Curitiba (PR), Brasil 
Leno Francisco Danner

Universidade Federal de Rondônia, Porto Velho (RO), Brasil

Leny Rodrigues Martins Teixeira

Universidade Estadual Paulista Júlio de Mesquita Filho, Presidente

Prudente (SP), Brasil

Lia Pinheiro Barbosa

Universidade Estadual do Ceará, Fortaleza (CE), Brasil

Lílian do Valle

Universidade Federal do Estado do Rio de Janeiro, Rio de Janeiro (RJ), Brasil

Livia Maria Fraga Vieira

Universidade Federal de Minas Gerais, Belo Horizonte (MG), Brasil

\section{Lluís Parerissa}

Universitat Autònoma de Barcelona, Barcelona, Espanha

Lourdes de Fátima Bezerra Carril

Universidade Federal de São Carlos, São Carlos (SP), Brasil

Lúcia Isabel Silva

Universidade Federal do Pará, Belém (PA), Brasil

Lúcia Maria de Assis

Universidade Federal Fluminense, Niterói (RJ), Brasil

Lúcia Mercês de Avelar

Universidade Estadual de Campinas, Campinas (SP), Brasil

Lucídio Bianchetti

Universidade Federal de Santa Catarina, Florianópolis (SC), Brasil

Lucila Maria Pesce de Oliveira

Universidade Federal de Santa Catarina, Florianópolis (SC), Brasil

Lucília Regina de Souza Machado

Centro Universitário UNA, Belo Horizonte (MG), Brasil

Lúcio França Teles

Universidade de Brasília, Brasília (DF), Brasil

Luís Antonio Groppo

Universidade Federal de Alfenas, Alfenas (MG), Brasil

Luís Miguel Carvalho

Universidade de Lisboa, Lisboa, Portugal

Luiz Antônio Cunha

Universidade Federal do Rio de Janeiro, Rio de Janeiro (RJ), Brasil

\section{Luiz Augusto Campos}

Universidade do Estado do Rio de Janeiro, Rio de Janeiro (RJ), Brasil
Luiz Fernandes Dourado

Universidade Federal de Goiás, Goiânia (GO), Brasil

Macarena Ruiz Balart

Universidad Carlos III de Madrid, Madrid, Espanha

Mailsa Passos

Universidade Federal do Estado do Rio de Janeiro, Rio de Janeiro (RJ), Brasil

Manoel Nelito Matheus Nascimento

Universidade Federal de São Carlos, São Carlos (SP), Brasil

Manuel Losada-Sierra

Universidad Militar Nueva Granada, Bogotá, Colômbia

Marcelo Henrique Romano Tragtenberg

Universidade Federal de Santa Catarina, Florianópolis (SC), Brasil

Marcelo Lima

Universidade Federal do Espírito Santo, Vitória (ES), Brasil

Marcelo Pinheiro Cigales

Universidade de Brasília, Brasília (DF), Brasil

Marcelo Siqueira Maia Vinagre Mocarzel

Universidade Federal Fluminense, Niterói (RJ), Brasil

Márcia Aparecida Jacomini

Universidade Federal de São Paulo, São Paulo (SP), Brasil

Marcia de Paula Leite

Universidade Estadual de Campinas, Campinas (SP), Brasil

Marco Antonio Espinoza Alvarado

Universidad de Chile, Santiago, Chile

Marcos Antônio Lorieri

Universidade Nove de Julho, São Paulo (SP), Brasil

Marcos Garcia Vidal

Universidad Nacional de Educación a Distancia, Madri, Espanha

Marcus Oliveira

Universidade Estadual Paulista Júlio de Mesquita Filho, Araraquara (SP), Brasil

Marcus Vinicius da Cunha

Universidade de São Paulo, São Paulo (SP), Brasil

María Begoña Alfageme González

Universidad de Murcia, Murcia, Espanha

Maria Cecília Luiz

Universidade Federal de São Carlos, São Carlos (SP), Brasil 


\section{Maria Clara Di Pierro}

Universidade de São Paulo, São Paulo (SP), Brasil

Maria Cristina Fernandes

Universidade Federal de São Carlos, São Carlos (SP), Brasil

Maria Dilneia Espíndola Fernandes

Universidade Federal de Mato Grosso do Sul, Campo Grande (MS), Brasil

\section{Maria Ferrer Ribot}

Universitat Autònoma de Barcelona, Barcelona, Espanha

\section{Maria Giudicissi Rehder}

Universidade de São Paulo, São Paulo (SP), Brasil

\section{Maria Graça Jacintho Setton}

Universidade Federal de São Paulo, São Paulo (SP), Brasil

\section{Maria Isabel da Cunha}

Universidade Católica de Pelotas, Pelotas (RS), Brasil

Maria Ivonete Barbosa Tamboril

Universidade Federal de Rondônia. Porto Velho (RO), Brasil

Maria João Cardona

Instituto Politécnico de Santarém, Santarém, Portugal

Maria Margarida Machado

Universidade Federal de Goiás, Goiânia (GO), Brasil

Maria Vieira Silva

Universidade Federal de Uberlândia, Uberlândia (MG), Brasil

\section{Mariana Calife Nóbrega}

Pontifícia Universidade Católica do Rio de Janeiro, Rio de Janeiro (RJ), Brasil

Mariane Campelo Koslinski

Universidade Federal do Rio de Janeiro, Rio de Janeiro (RJ), Brasil

Marilda de Oliveira Costa

Universidade do Estado de Mato Grosso, Cáceres (MT), Brasil

Marilda Pasqual Schneider

Universidade do Oeste de Santa Catarina, Joaçaba (SC), Brasil

\section{Marília Pinto de Carvalho}

Universidade de São Paulo, São Paulo (SP), Brasil

\section{Marília Pontes Sposito}

Universidade de São Paulo, São Paulo (SP), Brasil

\section{Mariona Massip}

Universitat Autònoma de Barcelona, Barcelona, Espanha
Marisa Fernanda da Silva Bueno

Universidade de Santa Cruz do Sul, Santa Cruz do Sul (RS), Brasil

Marise Nogueira Ramos

Fundação Oswaldo Cruz, Rio de Janeiro (RJ), Brasil

Marlene de Araújo

Centro Universitário do Leste de Minas Gerais, Coronel Fabriciano (MG), Brasil

Marta Belmar

Universidad Católica del Maule, Talca, Chile

Marta Camarero Figuerola

Universitat Rovira i Virgili, Tarragona, Espanha

Marta Comas

Consorci d'Educació de Barcelona, Barcelona, Espanha

Mauro Titton

Universidade Federal de Santa Catarina, Florianópolis (SC), Brasil

Miriam Abramovay

Faculdade Latino-Americana de Ciências Sociais. Ciudad de Guatemala, Guatemala

Miriam Fábia Alves

Universidade Federal de Goiás, Goiânia (GO), Brasil

Mônica Carvalho Magalhães Kassar

Universidade Federal de Mato Grosso do Sul, Campo Grande (MS), Brasil

\section{Mônica Castagna Molina}

Universidade de Brasília, Brasília (DF), Brasil

Myriam Inés Feldfeber

Universidad de Buenos Aires, Buenos Aires, Argentina

\section{Nadia Drabach}

Instituto Federal de São Paulo, São Paulo (SP), Brasil

\section{Nadja Hermann}

Pontifícia Universidade Católica do Rio Grande do Sul, Porto Alegre (RS), Brasil

\section{Nalú Farenzena}

Universidade Federal do Rio Grande do Sul, Porto Alegre (RS), Brasil

\section{Nathalia Cassetari}

Universidade de Brasília, Brasília (DF), Brasil

Nelson Rosário de Souza

Universidade Federal do Paraná, Curitiba (PR), Brasil 
Nilson Marcos Dias Garcia

Universidade Tecnológica Federal do Paraná, Curitiba (PR), Brasil

Nubia Regina Moreira

Universidade Estadual do Sudoeste da Bahia, Vitoria da Conquista (BA), Brasil

Nora Krawczyk

Universidade Estadual de Campinas, Campinas (SP), Brasil

Nydia NinnaValencia Jimenez

Universidad de Córdoba, Córdoba, Colômbia

Oscar Navajas Corral

Univerdidad de Alcalá de Henares, Madrid, Espanha

Otaviano Augusto Marcondes Helene

Universidade de São Paulo, São Paulo (SP), Brasil

Paulo Alberto dos Santos Vieira

Universidade do Estado de Mato Grosso, Cáceres (MS), Brasil

Pedro Angelo Pagni

Universidade Estadual Paulista Júlio de Mesquita Filho, Marília (SP), Brasil

Paulo Henrique Arcas

Universidade Federal de Lavras, Lavras (MG), Brasil

Rafael Arredondo Quijada

Universidad de Málaga, Málaga, Espanha

Raquel Fontes Borghi

Universidade Estadual Paulista Júlio de Mesquista Filho, Araraquara

(SP), Brasil

Regimeire Oliveira Maciel

Universidade Federal do ABC, Santo André (SP), Brasil

\section{Regina Tereza Cestari de Oliveira}

Universidade Católica Dom Bosco, Campo Grande (MS), Brasil

\section{Ricardo Alvarez}

Universidad Austral, Valdivia, Chile

\section{Ricardo Cuenca}

Instituto de Estudios Peruanos, Lima, Perú

\section{Roberto Leher}

Universidade Federal do Rio de Janeiro, Rio de Janeiro (RJ), Brasil

\section{Rodrigo Travitzki}

Universidade de São Paulo, São Paulo (SP), Brasil

Rogério de Almeida

Universidade de São Paulo, São Paulo (SP), Brasil
Ronaldo Marcos de Lima Araujo

Universidade Federal do Pará, Belém (PA), Brasil

Roque Rodrigues

Centro de Estudos Interdisciplinares em Educação e Desenvolvimento, Lisboa, Portugal

Rosa Maria Bueno Fischer

Universidade Federal do Rio Grande do Sul, Porto Alegre (RS), Brasil

Rosa Virgínia Wanderley Diniz

Universidade de Sorocaba, Sorocaba (SP), Brasil

Rosana Evangelista Cruz

Universidade Federal do Piauí, Teresina (PI), Brasil

Rosana Rodrigues Heringer

Universidade Federal do Rio de Janeiro, Rio de Janeiro (RJ), Brasil

Roseli Rodrigues de Mello

Universidade Federal de São Carlos, São Carlos (SP), Brasil

\section{Roser Calaf}

Universidad de Oviedo, Oviedo, Espanha

Rubens Barbosa de Camargo

Universidade de São Paulo, São Paulo (SP), Brasil

Rui Silva

Centro de Estudos Africanos da Universidade do Porto, Porto, Portugal

Ruy de Deus e Mello Neto

Universidade Federal do Ceará, Fortaleza (CE), Brasil

Saulo Rodrigues Carvalho

Universidade Estadual do Centro-Oeste, Guarapuava (PR), Brasil

Sebastian Donoso Diáz

Universidad de Talca, Talca, Chile

Selma Borghi Venco

Universidade Estadual de Campinas, Campinas (SP), Brasil

Sergio Stoco

Universidade Estadual de Campinas, Campinas (SP), Brasil

Sérgio Luiz Alves da Rocha

Instituto Federal de Educação, Ciência e Tecnologia do Rio de Janeiro, Rio de Jnaeiro (RJ), Brasil

Sofia Marin

Universidad de Valladolid, Valladolid, Espanha

Solange Martins Oliveira Magalhães

Universidade Federal de Goiás, Goiânia (GO), Brasil

Silke Weber

Universidade Federal de Pernambuco, Recife (PE), Brasil 
Silvana Aparecida de Souza

Universidade Estadual do Oeste do Paraná, Foz do Iguaçu (PR), Brasil

Silvio Gallo

Universidade Estadual de Campinas, Campinas (SP), Brasil

Silvio Ricardo Gomes Carneiro

Universidade Federal do ABC, Santo André (SP), Brasil

Tânia de Freitas Resende

Universidade Federal de Minas Gerais, Belo Horizonte (MG), Brasil

\section{Tatiane Cosentino Rodrigues}

Universidade Federal de São Carlos, São Carlos (SP), Brasil

Teise Oliveira Guaranha Garcia

Universidade de São Paulo, Ribeirão Preto (SP), Brasil

Thiago Alves

Universidade Federal de Goiás, Goiânia (GO), Brasil

Toni Reis

Universidade do Vale do Rio dos Sinos, São Leopoldo (RS), Brasil

Valdemar Sguissardi

Universidade Metodista de Piracicaba, Piracicaba (SP), Brasil

\section{Valeria Pilão}

Universidade Tuiuti do Paraná, Curitiba (PR), Brasil

Valeria Modesta Herrera Fernández

Universidad de Chile, Santiago, Chile

Vanda Lúcia Praxedes

Universidade do Estado de Minas Gerais, Divinópolis (MG) Brasil

\section{Vanessa Gabassa}

Universidade Federal de Goiás, Goiânia (GO), Brasil

Vera Lúcia Jacob Chaves

Universidade Federal do Pará, Belém (PA), Brasil

\section{Vera Maria Peroni}

Universidade Federal do Rio Grande do Sul, Porto Alegre (RS), Brasil

Vera Valdemarin

Universidade Estadual Paulista Júlio de Mesquita Filho, Araraquara (SP),

Brasil

\section{Vicente Riccio}

Universidade Federal de Juiz de Fora, Juiz de Fora (MG), Brasil

Vinícius Parolin Wohnrath

Universidade Estadual de Campinas, Campinas (SP), Brasil

\section{Virginia Gamez}

Universidad Nacional de Educación, Chuquipata Centro, Equador

Vivian Batista da Silva

Universidade de São Paulo, São Paulo (SP), Brasil

Viviane Ines Weschenfelder

Universidade do Vale do Rio dos Sinos, São Leopoldo (RS), Brasil

Wagner Wey Moreira

Universidade Federal do Triangulo Mineiro, Uberaba (MG), Brasil

Wenceslao Oliveira

Universidade Estadual de Campinas, Campinas (SP), Brasil

Wilma Baía Coelho

Universidade Federal do Pará, Belém (PA), Brasil

Zaida García Valecillo

Universidad Central de Venezuela, Caracas, Venezuela

Zara Figueiredo Tripodi

Universidade Federal de Ouro Preto, Ouro Preto (MG), Brasil 